\title{
O papel do cirurgião-dentista do trabalho no contexto das políticas públicas em saúde do trabalhador: artigo de revisão
}

\author{
The role of the occupational dentist in the context of public policies \\ for workers' health: review article
}

Jacira Pereira Dantas*

Flávia Godinho Costa Wanderley**

Ricardo Araújo da Silva**

Tatiana Frederico de Almeida ${ }^{* * * *}$

Urbino da Rocha Tunes ${ }^{* * * *}$

\section{Resumo}

Objetivo: realizar uma revisão de literatura sobre o papel do cirurgião-dentista do trabalho no contexto das políticas públicas no âmbito da Saúde do Trabalhador no Brasil. Métodos: neste estudo de cunho bibliográfico, foi feita uma busca com os seguintes descritores: saúde do trabalhador, odontologia do trabalho, saúde bucal do trabalhador e com os respectivos correspondentes em inglês: occupational dentistry, occupational health e worker oral health, nas bases de dados da Biblioteca Virtual em Saúde (BVS), com destaque para Scielo, Lilacs e BBO durante o período de 2002 a 2013. Resultados: o presente trabalho trouxe uma discussão voltada para três categorias temáticas. Categoria 1- Problemas odontológicos/condições de saúde bucal dos trabalhadores, Categoria 2- Absenteísmo por causas odontológicas e Categoria 3- Conhecimento da especialidade Odontologia do Trabalho e reconhecimento da importância da saúde bucal dos trabalhadores. Considerações finais: percebe-se, ao longo dos anos, que embora tenham ocorrido esforços em regulamentar políticas públicas voltadas à saúde bucal, não se tem contemplado a saúde bucal devidamente com a atuação profissional do cirurgião-dentista do trabalho e sua equipe.

Palavras-chave: Saúde do trabalhador. Odontologia do trabalho. Saúde bucal.

\section{Introdução}

Mudanças no modo de organizar e de gerir o trabalho têm gerado consequências substanciais para o processo de adoecimento das pessoas. Nessa perspectiva, as ações de segurança e saúde do trabalhador exigem uma atuação multiprofissional, interdisciplinar e intersetorial capaz de contemplar a complexidade das relações produção-consumo-ambiente e saúde. Nessa lógica, compreendendo que a saúde bucal não pode ser dissociada da saúde

Graduada em Odontologia pela Escola Bahiana de Medicina e Saúde Pública, Salvador, Bahia, Brasil. Residente em Saúde Coletiva pela Universidade do Estado da Bahia.

Estudante de Iniciação Científica da Escola Bahiana de Medicina e Saúde Pública, Salvador, Bahia, Brasil/ Bolsista FAPESB.

Mestre em Deontologia e Odontologia Legal pela Universidade de São Paulo, professor Assistente do Curso de Odontologia da Escola Bahiana de Medicina e Saúde Pública, Salvador, Bahia, Brasil.

**** Doutora em Saúde Coletiva pela Universidade Federal da Bahia, professora adjunta do Curso de Odontologia da Escola Bahiana de Medicina e Saúde Pública, Salvador, Bahia, Brasil.

Doutor em Imunologia pelo Instituto de Ciências da Saúde. Coordenador do Curso de Odontologia da Escola Bahiana de Medicina e Saúde Pública, Salvador, Bahia, Brasil. 
geral, faz-se necessário discutir a inclusão do cirurgião-dentista do trabalho no escopo das políticas públicas voltadas à saúde do trabalhador ${ }^{1}$.

Após quase dez anos de implantação das Diretrizes da Política Nacional de Saúde Bucal, implementadas em 2004, ainda são muitas as lacunas existentes no desenvolvimento de políticas que assessoram a saúde bucal do trabalhador no que tange aos agravos e aos riscos ocupacionais envolvendo a cavidade bucal. Com isso, o trabalhador continua marginalizado quanto ao acesso à política de promoção da saúde bucal ${ }^{2}$.

O desenrolar dessa temática necessariamente implica em trazer questionamentos acerca dos aspectos normativos que norteiam as ações, competências e responsabilidades, tanto no que se refere à legislação sanitária e trabalhista no campo da Saúde do Trabalhador, quanto aos instrumentos que dispõem sobre a área da higiene ocupacional e sobre as atribuições previstas para o cirurgião-dentista do trabalho.

Segundo Pizzato ${ }^{3}$ (2002), o modelo atual de organização da saúde do trabalhador está fortemente centralizado nos serviços médicos de empresa. Porém, esses serviços funcionam, em sua maioria, de forma autônoma e dissociada tanto do sistema de saúde, como dos órgãos regionais do Ministério do Trabalho.

Ademais, é sabido que o cirurgião-dentista que atua no Sistema Único de Saúde (SUS) trabalha numa lógica diferente daquele que está inserido no Programa de Saúde Ocupacional das empresas privadas. Contudo, vale ressaltar que os Serviços Especializados em Engenharia de Segurança e em Medicina do Trabalho (Sesmt), embora sejam normatizados pela legislação trabalhista, estão subordinados à legislação sanitária como se observa no artigo $6^{\circ}$, parágrafo $3^{\circ}$, inciso VI da Lei Orgânica do $\mathrm{SUS}^{4}$.

O objetivo deste trabalho é fazer uma revisão de literatura sobre o papel do cirurgião-dentista do trabalho no contexto das políticas públicas no âmbito da Saúde do Trabalhador.

\section{Políticas públicas e o campo da saúde do traballhador}

Diante das reivindicações advindas do cenário degradante das condições de trabalho imposto pelo capital e decorrentes de agravos que vitimavam os trabalhadores a partir de meados do século XIX, houve a necessidade de legitimação dos Direitos Sociais. Assim, o campo da Saúde do Trabalhador (ST) passa a ser palco de intervenções, bem como alvo de ações do Estado por meio da formulação de normas sanitárias, trabalhistas e de saúde e segurança do trabalho ${ }^{5}$.

As políticas públicas no Brasil, até 1988, caracterizavam-se pela proteção da saúde dos trabalha- dores de forma tutelada pelas áreas governamentais da Previdência Social e do Trabalho, por meio de uma política de Estado compensatória voltada aos trabalhadores constituintes, formalmente inseridos no mercado de trabalho. A saúde era apenas um benefício previdenciário para os contribuintes ou um serviço comprado na forma de assistência médica ou, por fim, uma ação de misericórdia oferecida aos que não tinham acesso à previdência e nem recursos para pagar a assistência privada, prestada por hospitais filantrópicos, como as Santas Casas ${ }^{1}$.

Assim, nos anos 1980 emergiram diversas mobilizações na tentativa de construção de um novo ideário para o campo da saúde do trabalhador. Uma delas incluiu a realização da I Conferência Nacional em Saúde do Trabalhador, em 1986, objetivando a incorporação da saúde do trabalhador no Sistema Único de Saúde ${ }^{6}$.

Nesse mesmo ano, a premissa exposta no Relatório Final da I Conferência Nacional de Saúde Bucal aponta a Saúde Bucal como sendo:

Parte integrante e inseparável da saúde do indivíduo, estando diretamente relacionada às condições de alimentação, moradia, trabalho, renda, meio ambiente, transporte, lazer, liberdade, acesso e posse de terra, acesso aos serviços de saúde e à informação.

Nesse sentido, a promulgação da Constituição Federal de 1988 (CF/88) vem assegurar os direitos fundamentais e prevê instrumentos garantidores das políticas públicas, entre os quais o Sistema Único de Saúde é o de maior predileção. Com esse novo ordenamento jurídico, o destaque deve ser dado ao modelo de saúde que emerge e amplia o olhar não só para ações predominantemente curativas, mas principalmente para as ações de prevenção. Reconhece, portanto, a essência coletiva do direito à saúde, condicionando sua garantia à execução de políticas públicas ${ }^{7}$.

Assim, as disposições normativas presentes na CF/88 subsidiaram a regulamentação do campo da saúde do trabalhador, em seu artigo $6^{\circ}$ parágrafo $3^{\circ}$, como sendo um conjunto de atividades que se destina, por meio das ações de vigilância epidemiológica e vigilância sanitária, à promoção e proteção da saúde dos trabalhadores, assim como visa à recuperação e reabilitação dos submetidos aos riscos e agravos advindos das condições de trabalho ${ }^{8}$.

Ainda, atendendo aos fins de proteção à saúde no ambiente laboral, a Lei Orgânica da Saúde, de 19 de setembro de 1990 , em seu artigo $6^{\circ}$, parágrafo $3^{\circ}$, regulamenta os dispositivos constitucionais referentes à saúde do trabalhador por meio de ações de vigilância epidemiológica e sanitária, de forma a garantir a promoção e proteção da saúde dos trabalhadores, bem como a recuperação e a reabilitação dos submetidos aos riscos e aos agravos advindos das condições de trabalho ${ }^{4}$.

A Portaria Ministerial nº 3.908, de 30 de outubro de 1998 estabelece procedimentos para orientar 
e instrumentalizar as ações e serviços de saúde do SUS, aprovando a Norma Operacional de Saúde do Trabalhador (NOST-SUS 98), com caráter complementar à Norma Operacional Básica 01/96 (NOB-SUS 01/1996). A manifestação vem assegurar que, todos os trabalhadores, independente de sua forma de inserção no mercado, devem ter acesso a todos os níveis de atenção à saúde, num movimento de integralidade, em que não só ações assistenciais devem ser providas, mas também ações de vigilância e promoção à saúde, regidas pelos mesmos princípios do SUS ${ }^{9}$.

$\mathrm{O}$ ordenamento jurídico nacional ainda prevê por meio da Consolidação das Leis do Trabalho, em seu título II, capítulo V, as diretrizes a serem seguidas pelo órgão fiscalizador do seu cumprimento, pelas empresas e pelos próprios empregados. Por certo, a proteção ao meio ambiente do trabalho é um direito assegurado aos trabalhadores, cabendo ao Poder Judiciário manter o cumprimento de normas de higiene e segurança do trabalho. Para tanto, por meio da Portaria no 3.214/1978 do Ministério do Trabalho foram regulamentadas as normas regulamentadoras, comumente conhecidas como NRs, relativas à Segurança e Medicina do Trabalho ${ }^{10}$.

Nesse sentido e considerando a necessidade de alinhamento entre a Política de Saúde do Trabalhador e a Política Nacional de Saúde dos Trabalhadores, a Portaria no 1.823 , de 23 de agosto de $2012^{11}$ institui a tão sonhada PNSST que, em seu artigo $6^{\circ}$, parágrafo único, dispõe que a realização da articulação tratada neste artigo requer mudanças substanciais nos processos de trabalho em saúde, na organização da rede de atenção e na atuação multiprofissional e interdisciplinar, que contemplem a complexidade das relações trabalho-saúde.

Ademais, a referida norma legal em seu artigo $8^{o}$, inciso III traz como um dos objetivos da Política Nacional de Saúde dos Trabalhadores garantir a integralidade na atenção à saúde do trabalhador, que pressupõe a inserção de ações de saúde do trabalhador em todas as instâncias e pontos de Rede de Atenção à Saúde do SUS, mediante articulação e construção conjunta de protocolos, linhas de cuidado e matriciamento da saúde do trabalhador na assistência e nas estratégias e dispositivos de organização e fluxos da rede, considerando os seguintes componentes: a) atenção primária em saúde; b) atenção especializada, incluindo serviços de reabilitação; c) atenção pré-hospitalar, de urgência e emergência, e hospitalar; d) rede de laboratórios e de serviços de apoio diagnóstico; e) assistência farmacêutica; f) sistemas de informações em saúde; g) sistema de regulação do acesso; h) sistema de planejamento, monitoramento e avaliação das ações; i) sistema de auditoria; j) promoção e vigilância à saúde, incluindo a vigilância à saúde do trabalhador.

\section{O Cirurgião-dentista na equipe de saúde e segurança do traballho}

Segundo Mazzili5 (2007), as Normas Regulamentadoras (NRs) são de observância obrigatória pelas empresas privadas e públicas e pelos órgãos públicos da administração direta e indireta, bem como pelos órgãos dos poderes Legislativo e Judiciário que empregam servidores regidos pela Consolidação das Leis do Trabalho - CLT. A legislação laboral obriga as empresas à criação de órgãos internos de proteção à saúde e à vida do trabalhador, a saber: Programa de Prevenção de Riscos Ambientais - PPRA, descrito na NR-9, Programa de Controle Médico de Saúde Ocupacional - PCMSO, previsto na NR-7, além da formulação do Perfil Profissiográfico Previdenciário - PPP ${ }^{10}$. Além disso, o Serviço Especializado em Engenharia de Segurança e em Medicina do Trabalho (SESMT), constante na NR-4, prevê classificação das atividades econômicas sob o critério do grau de risco ocupacional e estabelece o enquadramento das empresas em categorias ${ }^{12}$.

A NR-7 estabelece a obrigatoriedade da elaboração e a implementação do PCMSO, preconizando a promoção e a preservação da saúde dos trabalhadores nas empresas do país. Além disso, a norma supracitada estabelece os parâmetros que determinam as hipóteses nas quais as empresas estão obrigadas a manter os profissionais de saúde do trabalho (NR-4), aponta os parâmetros que facultam à empresa a indicação de um médico para coordenar o PCMSO, define competências médicas do coordenador e da equipe de saúde e elenca os exames obrigatórios. A NR-7, que se encontra defasada, deve prever a inclusão da Odontologia no PCMSO, pois além de atestar a saúde bucal dentro do sistema de saúde ocupacional, criar-se-ia um banco de dados para a área odontológica ${ }^{13}$.

Silva $^{2}$ (2012) aponta a necessidade de revisão das normas regulamentadoras e a importância da atuação do médico do trabalho dentro da Equipe de Saúde e Segurança. No entanto, chama a atenção para que a competência da cavidade bucal e dos agravos existentes e relacionados ao trabalho nessa área sejam de um especialista em saúde bucal do trabalhador. Assim, compete ao cirurgião-dentista fornecer o parecer técnico concernente à tais situações, verificando o nexo de causalidade existente entre essas morbidades e o ambiente laboral.

Nessa linha, esforços já vinham sendo feitos pelo Conselho Federal de Odontologia quanto à inserção da Odontologia no Programa de Controle Médico em Saúde Ocupacional da Secretaria de Segurança e Saúde do Trabalho, do Ministério do Trabalho.

O Conselho Federal de Odontologia já previa, em 2001, mediante a Resolução CFO nº 22, art. 30, seção X, título $\mathrm{I}^{14}$, que a Odontologia do Trabalho tem por objetivo a busca permanente da compatibilidade entre a atividade laboral e a preservação da saúde bucal do trabalhador. Compreende o estudo 
dos fenômenos físicos, químicos, biológicos e mecânicos que atingem o complexo bucomaxilofacial, e que podem influenciar ou ser influenciados pelo ambiente de trabalho.

Para tanto, foram definidas as seguintes áreas de competência da especialidade: identificação, avaliação e vigilância dos fatores ambientais que possam constituir risco à saúde bucal no local de trabalho, em qualquer das fases do processo de produção; assessoramento técnico e atenção em matéria de saúde, de segurança, de ergonomia e de higiene no trabalho, assim como em matéria de equipamentos de proteção individual, entendendo-se inserido na equipe interdisciplinar de saúde do trabalho operante; planejamento e implantação de campanhas e programas de duração permanente para educação dos trabalhadores quanto a acidentes de trabalho, doenças ocupacionais e educação em saúde; organização das estatística de morbidade e de mortalidade com causa bucal e investigação de suas possíveis relações com as atividades laborais; por fim, a realização de exames odontológicos para fins trabalhistas ${ }^{15}$.

Recentemente um projeto de lei (PL 422/2007) ${ }^{16}$ altera o art. 162, seção III e o art. 68 , seção V do título II da Consolidação das Leis do Trabalho (CLT - Decreto-Lei 5.452/1943) ${ }^{17}$, para obrigar as empresas a manter serviços especializados em segurança em Medicina e em Odontologia do Trabalho. Desse modo, será obrigatória a participação do dentista na equipe de saúde do trabalhador e também será obrigatória a realização dos exames admissionais e pré-admissionais, dentre outros. Se aprovado, as empresas terão cerca de 360 dias para tomar providências e colocar em prática as novas medidas.

\section{Métodos}

Trata-se de uma pesquisa de cunho bibliográfico, constituindo uma revisão de literatura referente ao papel do cirurgião-dentista especialista em saúde do trabalhador no contexto das políticas públicas.

Apenas um avaliador, previamente calibrado, realizou o levantamento na literatura em geral, a partir das fontes primárias: livros, leis e portarias federais, textos do Ministério da Saúde, notas técnicas, notícias veiculadas em informativos do conselhos federal e regional de Odontologia da Bahia e da Associação Brasileira de Odontologia, relatórios de conferências e encontros regionais sobre saúde do trabalhador.

Como estratégia de busca, foram pesquisados os seguintes descritores: saúde do trabalhador, odontologia do trabalho, saúde bucal do trabalhador com os respectivos correspondentes em inglês: occupational dentistry, occupational health e worker oral health, nas bases de dados da Biblioteca Virtual em Saúde (BVS), com destaque para Scielo, BBO e Lilacs, durante o período de junho a dezembro de 2013. Foram realizados os seguintes cruzamentos entre os des- critores: "política pública" e "saúde do trabalhador"; "Saúde trabalhador" e "odontologia do trabalho"; "política pública" e "odontologia do trabalho", bem como o cruzamento entre os três termos e seus respectivos sinônimos e correspondentes em inglês.

Os critérios de inclusão adotados para seleção dos artigos foram: publicações compreendidas no período de 2002 a 2013, lusofônicas e que estivessem disponíveis na íntegra nas bases de dados selecionadas, que abordassem políticas públicas e a inserção da Odontologia do Trabalho. O critério de exclusão adotado para este estudo pautou-se na eliminação de todos os casos que apresentavam esses descritores, porém não abrangiam o período de 2002 a 2013, e não se referissem à relação entre políticas públicas e Odontologia do Trabalho.

Como o reconhecimento da especialidade Odontologia do Trabalho pelo Conselho Federal de Odontologia foi em 2002, poucos trabalhos foram publicados no período de 2002 a 2013 com os descritores supracitados. Dessa forma, esse fator implicou fortemente na compilação de trabalhos para este estudo.

$\mathrm{Na}$ BVS, após cruzamento entre os descritores "saúde do trabalhador" (occupational health) e "política de saúde" (health policy) foram encontradas 951 publicações. Após considerar os critérios de inclusão e de exclusão foram selecionados treze textos completos. Ao utilizar os termos "Odontologia do Trabalho" (occupational dentistry) e Saúde do trabalhador (occupational health) obteve-se 26 publicações. Não foram encontrados trabalhos no cruzamento entre os descritores "Odontologia do Trabalho (occupational dentistry) e "política de saúde" (health policy). Após realizar busca com os três termos acima mencionados, foi encontrado um artigo de revisão.

Uma vez que a especialidade Odontologia do Trabalho é recente e, portanto, ainda carece de mais publicações na área, muitos artigos não traziam o método de estudo adotado, constituindo ensaios de revisão, fato que inviabiliza uma melhor sistematização dos trabalhos existentes. Dessa forma, foi utilizada a revisão de literatura narrativa, em detrimento da revisão sistemática.

\section{Resultados e discussão}

Após levantamento bibliográfico, os dados foram analisados de acordo com a Técnica de Análise Temática de Minayo que prevê a formação de categorias para que se estabeleçam classificações, ou seja, o agrupamento de ideias, elementos ou expressões em torno de um conceito ${ }^{18}$.

Assim, a partir de uma leitura cuidadosa e da reflexão sobre o objeto de estudo e suas interfaces, buscou-se uma aproximação entre a temática e a literatura encontrada, o que culminou na formação de categorias. Desse modo, o presente trabalho tra- 
rá uma discussão voltada para três categorias temáticas de acordo com os resultados encontrados, a saber: Categoria 1 - Problemas odontológicos/condições de saúde bucal dos trabalhadores, Categoria 2 - Absenteísmo por causas odontológicas e Categoria 3 - Conhecimento da especialidade Odontologia do Trabalho e reconhecimento da importância da saúde bucal dos trabalhadores. Tais categorias foram discutidas à luz da literatura disponível sobre o tema em estudo, considerando livros, artigos científicos e dissertações.

\section{Categoria 1. Problemas odontológicos/ condições de saúde bucal}

No que concerne aos problemas dentários e ou às condições de saúde bucal, é sabido que no Brasil, onde existe grande disparidade sócioeconômico e limitada atenção das políticas públicas à saúde bucal dos adultos, tornam-se alarmantes os indicadores de saúde bucal dessa população economicamente ativa, corroborando com o elevado CPO-D encontrado, o que expressa a alta prevalência de cárie dentária, expressivo número de dentes perdidos e comprometimento periodontal, além da grande necessidade do uso de próteses, em conformidade com o estudo de Tauchen ${ }^{19}(2006)$.

Segundo Almeida e Vianna ${ }^{20}(2005)$ existem relatos de associação potencial entre exposições ocupacionais e alterações bucais, destacando a importância dos dados epidemiológicos no planejamento de programas de saúde bucal do trabalhador. Dentre os estudos analisados pelas autoras, existe maior predominância de pesquisas sobre substâncias ácidas e exposições relacionadas com o açúcar. No entanto, percebe-se que os programas de saúde bucal não levam em consideração as especificidades dos riscos dos trabalhadores expostos tanto a esses fatores quanto aos demais inerentes do ambiente de trabalho.

Ademais, deve-se atentar ao perfil da população adulta economicamente ativa, vulnerável aos fatores de risco no ambiente laboral, bem como aos fatores etiológicos comuns relacionados às doenças bucais ${ }^{20}$.

Outro ponto a ser discutido diz respeito ao Serviço Especializado de Odontologia do Trabalho que tem sido ofertado pelas empresas, seja como integrante de um Programa de Saúde e Segurança do Trabalho da própria instituição ou oferecido de forma conveniada. A odontologia de grupos ou empresarial caracteriza-se pela venda de certo trabalho odontológico por um valor previamente estipulado e de acordo com determinados padrões acordados pelos empregadores. Nesse caso, o cirurgião-dentista atuará conforme as normas estabelecidas pelo contratante ${ }^{13}$. Verifica-se por meio dos estudos que algumas empresas ainda trazem em seu quadro aspectos de uma odontologia com ênfase apenas na prática curativa.
No estudo de Pizzato e Garbin ${ }^{21}$ (2006), observou-se a fragilidade dos serviços ofertados em Odontologia do Trabalho ao se estudar a existência de programa de atenção em saúde bucal em indústrias do setor privado dos municípios de Araçatuba e Birigui (SP). A assistência odontológica era oferecida em apenas $30 \%$ das empresas avaliadas, nas quais predominava a prática curativo-restauradora, com pouca ênfase em atividades preventivas.

Gomes e $\operatorname{Abegg}^{22}$ (2007) verificaram que a maioria dos problemas bucais afeta sobremaneira o desempenho diário dos trabalhadores.

\section{Categoria 2. Absenteísmo por causas odontológicas}

No que tange ao absenteísmo por causas odontológicas, Coelho et al. ${ }^{23}$ (2010) aponta em seu estudo, que $33 \%$ dos absenteísmos foram causados por motivos odontológicos. As causas identificadas foram: consulta regular para tratamento (20\%), doença gengival (4\%), confecção de prótese (2\%), seguida de consulta para prevenção e exodontia, com $1 \%$ em cada caso. Tal estudo corrobora os achados de Silva $^{2}$ (2012), ao afirmar que o absenteísmo ao trabalho por situações referentes às morbidades que acometem a cavidade bucal é salientado por muitos autores e verificado em diversos estudos epidemiológicos chegando, em média, a $10 \%$ das faltas ao trabalho.

No entanto, no estudo de Martins et al. ${ }^{24}$ (2005) o absenteísmo por motivos odontológicos não foi tão relevante se comparado ao total de faltas ao trabalho por motivo de doença, além do afastamento do trabalhador ocorrer em um período menor.

Santos et al. ${ }^{25}$ (2008) estudou o absenteísmo de funcionários em uma empresa que havia implantado o serviço de assistência odontológica, enfatizando o número de atestados fornecidos pelo consultório odontológico do Sesmt. Em 1995, foram emitidos, pela equipe de odontologia do Sesmt, 170 atestados. Em 2006, esse número reduziu para 99. Com relação à troca de atestados externos avalizados pela equipe, o número caiu de 177 , em 1995, para sete, em 2006, o que sugere a importância da atuação do cirurgião-dentista na redução do absenteísmo por causas odontológicas.

\section{Categoria 3. Conhecimento da especialidade Odontología do Traballho e reconhecimento da importância da saúde bucal dos trabalhadores}

No que tange ao conhecimento da especialidade Odontologia do Trabalho e ao reconhecimento da importância da saúde bucal dos trabalhadores, verifica-se que ainda há muito desconhecimento 
por parte dos profissionais das empresas sobre a referida área. Hiroishi et al. ${ }^{26}$ (2011) asseveram tal afirmativa salientando que apesar de se estudar há muito tempo essa relação, ainda existe pouco conhecimento por parte da equipe de saúde bucal à respeito do nexo causal entre o ambiente de trabalho e as consequências na cavidade bucal.

$\operatorname{Costa}^{27}$ (2005) apresentou uma revisão de literatura sobre a nova especialidade Odontologia do Trabalho e relatou a dificuldade encontrada na busca por artigos de revistas especializadas nessa área, uma vez que as universidades ainda não produzem ciência nessa nova área, mas, sim, na área de saúde ocupacional, em consonância com os achados do presente trabalho.

Diante do exposto nas categorias supracitadas, parece claro que o cirurgião-dentista tem uma grande responsabilidade na detecção e, consequentemente, na prevenção das doenças profissionais que possam ser diagnosticadas por meio do exame da cavidade bucal ${ }^{28}$. Quando se reconhece o indivíduo como um todo e não como um ser fragmentado coaduna-se com uma visão da saúde bucal de forma mais holística e atual.

Entende-se que a implantação de um programa de saúde bucal nas empresas deve preconizar a melhora das condições de saúde do trabalhador baseado em princípios éticos e humanísticos, respeitando sua condição de ser humano, e não simplesmente como peças de um mecanismo de produção ${ }^{3}$.

Como dito, o reconhecimento da necessidade de implantação dos serviços odontológicos e, consequentemente, da integração do cirurgião-dentista nas equipes de saúde e segurança do trabalho, bem como seu papel justifica-se, também, pela alta prevalência de problemas relacionados à cárie dentária e doenças periodontais, a possibilidade de detecção precoce do câncer bucal, além das manifestações bucais da Aids. Podem ocorrer acidentes de trabalho no ambiente laboral, envolvendo estruturas bucais ou manifestações de doenças ocupacionais bucais com comprometimento sistêmico.

Em síntese, torna-se evidente a necessidade de inserção do cirurgião-dentista do trabalho em órgãos internos de proteção à saúde e à vida do trabalhador, a saber: Programa de Prevenção de Riscos Ambientais, bem como no Programa de Controle Médico de Saúde Ocupacional, de modo a superar a lacuna existente no que diz respeito à promoção, à proteção e à recuperação dos agravos causados por agentes etiológicos relacionados às doenças do sistema estomatognático. Tauchen ${ }^{19}(2006)$ aponta a necessidade de ampliação do Programa de Saúde Ocupacional das empresas, estendendo a equipe do Sesmt com a inclusão do cirurgião-dentista do trabalho. Entende-se que esse profissional possa atuar na realização de levantamentos epidemiológicos, possibilitando a detecção dos focos de infecção que, porventura, venham causar dor ou qualquer desconforto, bem como no absenteísmo por doenças profissionais.

\section{Considerações finais}

Percebe-se ao longo dos anos que embora tenham ocorrido esforços em regulamentar políticas públicas voltadas à saúde bucal, não tem sido devidamente contemplada a saúde bucal do trabalhador, no que diz respeito à à atuação profissional do cirurgião-dentista do trabalho e sua equipe. É sabido que há uma lacuna na assistência aos trabalhadores nas Unidades de Saúde da Estratégia de Saúde da Família, visto que o funcionamento do Programa atende em horário comercial, momento em que a classe economicamente ativa encontra-se em seus locais de trabalho.

Por fim, do ponto de vista metodológico, esta é uma revisão de literatura narrativa, convencional. Seus achados podem ser enviesados, uma vez que não foram analisados segundo critérios avaliativos de uma escala qualitativa ou quantitativa, conforme preconiza uma revisão da literatura sistemática. Seus achados, entretanto, apontam que, após dez anos de regulamentação da especialidade Odontologia do Trabalho, a área ainda carece de mais estudos que subsidiem melhor o papel do cirurgião-dentista na Saúde do Trabalhador. Os achados ainda apontam para uma Odontologia Ocupacional, em que predomina uma atuação ainda assistencialista do cirurgião-dentista. Por fim, salienta-se que o papel do cirurgião dentista do trabalho deve ser pautado na identificação, na avaliação e na vigilância dos fatores ambientais que possam constituir risco à saúde bucal do trabalhador. Sua prática deve comungar com os princípios da atual Política $\mathrm{Na}$ cional de Saúde dos Trabalhadores, considerando a transversalidade das ações no campo da saúde do trabalhador e enfatizando o trabalho como um dos determinantes do processo saúde-doença.

\section{Abstract}

Objective: To perform a literature review on the role of the occupational dentist in the context of public policies, focusing on workers' health. Materials and method: In this bibliographic study, a search was made with the following descriptors and their corresponding English terms: workers' health, occupational dentistry, and workers' oral health, in the databases of the Health Virtual Library (BVS - Scielo, Lilacs and BBO) during the period from 2002 to 2013. Results: The present work discussed three thematic categories, namely: Category 1- Dental problems/oral health conditions of workers, Category 2- Absenteeism by dental causes, and Category 3- Knowledge of the specialty of occupational dentistry and recognition of the importance of workers' oral health. Final considerations: It is noted, over the years, that although there have been efforts to regulate 
public policies for oral health, workers' oral health has not been properly dealt with the professional practice of occupational dental surgeons and their teams.

Keywords: Occupational health. Occupational dentistry. Oral health.

\section{Referências}

1. Brasil. Ministério da Saúde. Legislação em saúde: caderno de legislação em saúde do trabalhador. Brasília; 2005.

2. Silva R. A necessidade de inserção do cirurgião-dentista na equipe de saúde e segurança do trabalho. Rev Conselho Regional de Odontologia da Bahia 2012;1(4):10-1.

3. Pizzato E. A saúde bucal no contexto da saúde do trabalhador: análise dos modelos de atenção [Dissertação de Mestrado]. Araçatuba: Faculdade de Odontologia da Universidade estadual Paulista; 2002.

4. Brasil. Lei n. 8.080/90, de 19 de setembro de 1990. Constituição da República Federativa do Brasil; Brasília.

5. Mazzili LEN. Odontologia do trabalho. 2. ed. São Paulo: Livraria Santos; 2007.

6. Brasil. Ministério da Saúde. Relatório da I Conferência Nacional de Saúde do Trabalhador. Brasília; 1986.

7. Bartolomei CEF, Carvalho MS, Delduque MC. A saúde é um direito? Saúde em Debate 2003;65:184-91.

8. Brasil. Constituição 1988, de 9 de novembro de 1985. República Federativa do Brasil; Brasília.

9. Chiavegatto CV. Percepção dos Profissionais de nível superior da atenção primária quanto ao desenvolvimento de ações de saúde do trabalhador no SUS em Minas Gerais [Dissertação de Mestrado]. Belo Horizonte: Faculdade de Medicina da Universidade Federal de Minas Gerais; 2010.

10. Júnior JC. Curso de direito do trabalho. 8. ed. Salvador: Juspodivm; 2012.

11. Brasil. Portaria $\mathrm{n}^{-} 1.823$, de 23 de agosto de 2012. Diário Oficial da República Federativa do Brasil; Brasília, 23 de ago 2012, seção 1, p. 46-51.

12. Vasconcelos MM, Queluz DP. Conhecimento sobre odontologia do trabalho dos profissionais integrantes da saúde ocupacional em empresas. Odonto 2010;18(36):7-16.

13. De Araújo ME, Júnior AG. Saúde bucal do trabalhador: os exames admissional e periódico como um sistema de informação em saúde. Odontologia e Sociedade 1999;1(1/2):15-8.

14. Conselho Federal de Odontologia. Resolução n. 22, de 27 dezembro de 2001. Diário Oficial da República Federativa do Brasil; Brasília, seção 10.

15. Conselho Federal de Odontologia. Resolução no ${ }^{\circ} 63$, de 8 de abril de 2005. Diário Oficial da União; Brasília, 19 de abril 2005, seção 1, p. 104.

16. Brasil. Lei n. 422 , de 7 de dezembro de 2007. Constituição da República Federativa do Brasil; Brasília.

17. Brasil. Lei n. 5.452, de 1 de maio de 1943. Constituição da República Federativa do Brasil; Brasília.

18. Minayo MCS. O Desafio do conhecimento: pesquisa qualitativa em saúde. 7. ed. São Paulo: Hucitec; 2000.

19. Tauchen ALO. A Contribuição da Odontologia do trabalho no Programa de Saúde Ocupacional: verificando as condições de saúde bucal de trabalhadores de uma agroindústria do sul do Brasil [Dissertação de Mestrado]. São Paulo: Faculdade de Odontologia da Universidade de São Paulo; 2006.
20. De Almeida TF, Vianna MIP. O Papel da epidemiologia no planejamento das ações de saúde bucal do trabalhador. Saúde e Sociedade 2005;14(3):144-54.

21. Pizzato E, Garbin CAS. Odontologia do trabalho: implantação da atenção em saúde bucal do trabalhador. Odontol Clín Cient 2006;5(2):99-102.

22. Gomes AS, Abegg C. O impacto odontológico no desempenho diário dos trabalhadores do Departamento Municipal de Limpeza Urbana de Porto Alegre, Rio Grande do Sul, Brasil. Cad Saúde Pública 2007;23(7):1707-14.

23. Coelho MP, Oliveira MA, De Araújo VE, Carvalho CM. Absenteísmo por causas odontológicas em uma empresa agropecuária da região sudeste do estado de Minas Gerais. Rev Bras de Pesquisa em Saúde 2010;12(1):14-8.

24. Martins RJ, Garbin CAS, Garbin AJI, Moimaz SAS. Absenteísmo por motivos odontológico e médico nos serviços público e privado. Rev Bras Saúde Ocup 2005;30(111):9-15.

25. Santos PSS, Pinto MF, Neto JAG. Odontologia do trabalho em ambiente hospitalar. Rev Odonto Ciênc 2008;23(3):307-10.

26. Hiroishi WK, Rosetti E, Orenha ES, Naressi SCM. Odontologia do trababalho: um novo olhar sobre a saúde bucal do trabalhador. Braz Dent Sci 2011;14(3/4):66-76.

27. Costa SS. Odontologia do trabalho: nova área de atuação. Rev Assoc Paul Cir Dent 2005;59(6):432-6.

28. Peres SHC, Theodoro DS, Ribeiro DA, De Ávila ED, Greghi GA, Da Silva RPR. Odontologia do trabalho: doenças e lesões na prática profissional. Rev Odontol de Araçatuba 2006;27(1):54-8.

\section{Endereço para correspondência:}

Tatiana Frederico de Almeida

Rua João Bião de Cerqueira 251, Pituba

41.830.580 Salvador-BA

Telefone: (71) 3351-4447/9933-4886

E-mail: tatifrederico@yahoo.com.br

Recebido: 05/11/2014. Aceito: 06/04/2015. 